\title{
Cuff-Based Oscillometric Measurements of Central Hemodynamics: Factors Influencing Central Augmentation Pressure in Normotensive Japanese Individuals
}

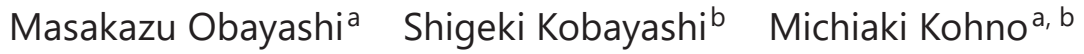 \\ Tomoko Nakashima $^{a}$ Masafumi Yano ${ }^{b}$ \\ a Department of Cardiovascular Medicine, Sanyo-Onoda City Hospital, Sanyo-Onoda, Japan; \\ ${ }^{b}$ Division of Cardiology, Department of Medicine and Clinical Science, Yamaguchi University \\ Graduate School of Medicine, Ube, Japan
}

\section{Keywords}

Augmentation pressure $\cdot$ Age $\cdot$ Asian population $\cdot$ Brachial oscillometry

\begin{abstract}
Background: Recently, new devices using oscillometric cuff have been developed to derive central blood pressure (BP) waveform from brachial BP waveform. Late systolic pressure augmentation of central BP waveform is a marker of central hemodynamics. Mobil-O-Graph, a cuff-based oscillometric device, can assess central augmentation pressure (AP) together with central BP. Central AP measurement using the Mobil-O-Graph in the European population was reported to be associated with age and sex. However, factors influencing central AP in the Asian population have not been shown. Objectives and Methods: We enrolled 110 normotensive volunteers (50 men; age range, 21-76 years). Central BP and AP were measured using the Mobil-O-Graph on the left arm with the subjects in the seated position after resting for at least $5 \mathrm{~min}$. We compared central hemodynamics between the sexes. We investigated factors influencing central AP in uni- and multivariate linear regression analyses and age-related change in central AP using the Mobil-O-Graph in healthy Japanese individuals. Results: Central AP were lower in men than in women $(5.5 \pm 2.8$ vs. $11.0 \pm 4.7 \mathrm{~mm} \mathrm{Hg}, p<$ 0.001). The central AP in the total cohort was positively correlated with age $(r=0.325, p<$ $0.001)$ and inversely correlated with height $(r=-0.601, p<0.001)$ in the Pearson correlations. In multivariate regression analysis, the parameters influencing central AP $\left(R^{2}=0.467\right)$ were age $(\beta=0.097, p<0.001)$, sex $(\beta=-2.890, p=0.010)$, and height $(\beta=-0.153, p=0.031)$. The
\end{abstract}


central AP $(10.0 \pm 4.8 \mathrm{~mm} \mathrm{Hg})$ in the $\geq 50$-year-old group significantly increased compared with those in the 20- to 39-year-old group $(6.7 \pm 4.2 \mathrm{~mm} \mathrm{Hg}, p<0.05)$. Conclusions: Age, sex, and height influenced central AP, as assessed using the Mobil-O-Graph. Age-related increase in central AP was observed in normotensive Japanese individuals. Brachial cuff-based waveform recordings using the Mobil-O-Graph are feasible for the estimation of central AP in the Asian population.

(c) 2019 S. Karger AG, Basel

\section{Introduction}

Central augmentation index (AIx) expresses late systolic pressure augmentation normalized by central pulse pressure. An increase in central AIx independently has been reported to correspond to a risk of increase in cardiovascular events and all-cause mortality [1]. Radial AIx is highly correlated with central AIx [2]. It has been reported that radial AIx is positively associated with age in Japanese individuals without cardiovascular disease [3] and that female sex and shorter height are important risk factors of elevated AIx in Asian patients with untreated hypertension, as assessed by using radial tonometry [4].

The ARCSolver method is used to measure central blood pressure (BP) based on the brachial BP waveform using a generalized transfer-function method [5]. The Mobil-O-Graph PWA monitor system (I.E.M. GmbH, Stolberg, Germany) is a device based on the ARCSolver transfer function and automatically measures central BP and central AIx using an oscillometric cuff [5]. The measurement of central AIx and augmentation pressure (AP) using the Mobil-O-Graph in the European population was reported to be associated with age and sex [6]. However, factors influencing central AIx and AP measured by using the Mobil-0-Graph in the Asian population have not been shown.

AIx increases steeply between the ages of 20 and 40 years and then plateaus after 60 years. The relationship between central AIx and age is nonlinear, whereas that between central AP and age is linear [6, 7]. Higher central AIx and central AP are associated with increased risk of development of coronary artery disease in younger and middle-aged men [8]. Here, we investigated factors influencing central AP in uni- and multivariate linear regression analyses and age-related change in central AP using the Mobil-0-Graph in healthy Japanese individuals.

\section{Methods}

Participants and Clinical Measurements

We recruited volunteers who understood the purpose of the study at Sanyo-Onoda City Hospital. All volunteers were nonsmokers, normotensive, and nondiabetic who were not taking any medications.

Demographic data, including age, height, weight, and sex, were obtained for each subject, whereas arm circumference was measured around the midpoint of the left upper arm. The measurements were obtained in a quiet and temperature-controlled $\left(22-25^{\circ} \mathrm{C}\right)$ room. The participants fasted for at least $4 \mathrm{~h}$ before the measurements, which were obtained after resting for at least $5 \mathrm{~min}$ in a seated position.

Tonometric Measurements of AIx

The left radial arterial waveform was recorded by applying the tonometric method using an automated radial pulse wave acquisition device HEM-9000AI (Omron Healthcare Co., Ltd., Kyoto, Japan). Radial AIx is the ratio of the amplitude of the late systolic peak to the amplitude of the early systolic peak and was automatically calculated using a fourth-order differential equation for radial arterial waveform [3]. 
Obayashi et al.: Augmentation Pressure by Brachial Oscillometry

Table 1. Clinical characteristics and hemodynamic parameters of the participants

\begin{tabular}{lcccr}
\hline & $\begin{array}{l}\text { Total } \\
(n=110)\end{array}$ & $\begin{array}{l}\text { Men } \\
(n=50)\end{array}$ & $\begin{array}{l}\text { Women } \\
(n=60)\end{array}$ & \multicolumn{1}{c}{$\begin{array}{l}p \text { value } \\
\text { (men vs. women) }\end{array}$} \\
\hline Age, years & $44.9 \pm 13.7$ & $44.1 \pm 14.0$ & $45.5 \pm 13.5$ & 0.609 \\
Height, cm & $163.1 \pm 10.1$ & $171.7 \pm 7.1$ & $156.0 \pm 5.3$ & $<0.001$ \\
Weight, kg & $58.4 \pm 11.0$ & $66.4 \pm 10.0$ & $51.8 \pm 6.6$ & $<0.001$ \\
HR, beats/min & $71.8 \pm 10.4$ & $69.2 \pm 9.8$ & $74.1 \pm 10.4$ & 0.012 \\
Brachial SBP, mm Hg & $117.2 \pm 10.2$ & $120.4 \pm 9.9$ & $114.4 \pm 9.7$ & 0.002 \\
Brachial DBP, mm Hg & $73.6 \pm 9.0$ & $78.8 \pm 7.7$ & $69.4 \pm 7.8$ & $<0.001$ \\
Brachial MBP, mm Hg & $93.5 \pm 9.0$ & $97.9 \pm 8.2$ & $89.9 \pm 8.1$ & $<0.001$ \\
Central SBP, mm Hg & $109.4 \pm 9.8$ & $112.2 \pm 9.8$ & $107.9 \pm 9.1$ & 0.002 \\
Central PP, mm Hg & $34.4 \pm 9.2$ & $32.1 \pm 6.0$ & $36.3 \pm 6.7$ & $<0.001$ \\
Central AIx, \% & $23.9 \pm 10.9$ & $16.8 \pm 7.3$ & $29.7 \pm 9.7$ & $<0.001$ \\
Central AP, mm Hg & $8.5 \pm 4.8$ & $5.5 \pm 2.8$ & $11.0 \pm 4.7$ & $<0.001$ \\
Radial AIx, \% & $77.2 \pm 15.1$ & $69.8 \pm 15.3$ & $83.4 \pm 12.0$ & $<0.001$ \\
aPWV, m/s & $6.7 \pm 1.6$ & $6.7 \pm 1.7$ & $6.7 \pm 1.5$ & 0.853 \\
\hline
\end{tabular}

Data are presented as mean \pm SD. $p$ values were calculated using an unpaired or Welch $t$ test to compare men and women. HR, heart rate; SBP, systolic blood pressure; DBP, diastolic blood pressure; MBP, mean blood pressure; PP, pulse pressure; AIx, augmentation index; AP, augmentation pressure; aPWV, aortic pulse wave velocity.

\section{Oscillometric Measurements of AIx and AP}

Following a break after the measurements using the HEM-9000AI, brachial and central BP measurements were performed on the left arm using the Mobil-O-Graph. Arm circumferences were measured and recorded to guide the correct choice of cuff (20-24 or 24-32 cm). The smaller cuff was used when the arm circumference was $24 \mathrm{~cm}$.

The Mobil-O-Graph had an inbuilt ARCSolver (Austrian Institute of Technology, Vienna, Austria). After brachial BP was measured, the cuff was instantly inflated, and brachial artery pressure waves were recorded, holding the diastolic BP level for $10 \mathrm{~s}$. Brachial artery pressure wave was digitized with a high-fidelity pressure sensor connected to a 12-bit A/D converter [5]. Central AIx and AP were calculated using a generalized transfer function $[5,6]$. The ARCSolver transfer function includes an algorithm for checking the signal quality. We analyzed only excellent or good-quality results, that is, $>80 \%$ or $>50 \%$ of signals used for the transfer function, respectively.

The subjects were divided into the following three age groups: $20-39$ years $(n=41 ; 22$ men), 40-49 years ( $n=27 ; 10$ men), and $\geq 50$ years ( $n=42 ; 18$ men). Age-related changes in radial AIx, central AIx, and central AP were assessed.

Estimates of aortic pulse wave velocity (aPWV) were derived using the ARCSolver method combined in a proprietary mathematical model. The major determinants included in this model were age, central pressure, and aortic characteristic impedance. A comparison of aPWV measured using invasive aortic pressure readings and the ARCSolver method showed a highly linear correlation $(r=0.81, p<0.0001)$ [9].

\section{Statistical Analysis}

Continuous data were expressed as mean \pm SD. The unpaired Student $t$ test or Welch $t$ test was used to analyze the differences between the sexes (men vs. women). Comparisons among the three age groups were performed using the Bartlett test. For the intergroup comparisons, the Bonferroni post hoc correction or the Kruskal-Wallis test was used. $p$ values $<0.05$ were considered significant.

Uni- and multivariate linear regression analyses were performed to assess the determinants of central AP. Pearson correlation coefficients between various parameters (age, height, weight, brachial mean BP, and heart rate) were calculated for central AP. Variables that were statistically significant in the univariate analysis were used in the multivariate analysis.

Analyses were performed using GraphPad Prism 6 (GraphPad Software Inc., La Jolla, CA, USA) and R version 3.2.3 (R Foundation for Statistical Computing, Vienna, Austria). Multivariate analysis was performed using $\mathrm{R}$ version 3.2.3. 
Table 2. Uni- and multivariate analyses of central augmentation pressure and other variables

\begin{tabular}{|c|c|c|c|c|c|}
\hline \multirow[t]{2}{*}{ Variables } & \multicolumn{2}{|c|}{ Univariate } & \multicolumn{3}{|c|}{ Multivariate } \\
\hline & $r$ & $p$ value & $\beta$ & standard error for $\beta$ & $p$ value \\
\hline \multicolumn{3}{|l|}{ Total $(n=110)$} & \multicolumn{3}{|c|}{$\left(R^{2}=0.467 ;\right.$ adjusted $\left.R^{2}=0.447\right)$} \\
\hline Age (years) & 0.325 & $<0.001$ & 0.097 & 0.025 & $<0.001$ \\
\hline Sex & - & - & -2.890 & 1.108 & 0.010 \\
\hline Height $(\mathrm{cm})$ & -0.601 & $<0.001$ & -0.153 & 0.070 & 0.031 \\
\hline Weight (kg) & -0.505 & $<0.001$ & -0.009 & 0.052 & 0.865 \\
\hline MBP (mm Hg) & -0.122 & 0.202 & - & - & - \\
\hline HR (beats/min) & 0.101 & 0.296 & - & - & - \\
\hline
\end{tabular}

$r$, Pearson correlation coefficient in univariate analysis; $\beta$, standardized coefficient in multivariate analysis; MBP, mean blood pressure; HR, heart rate.

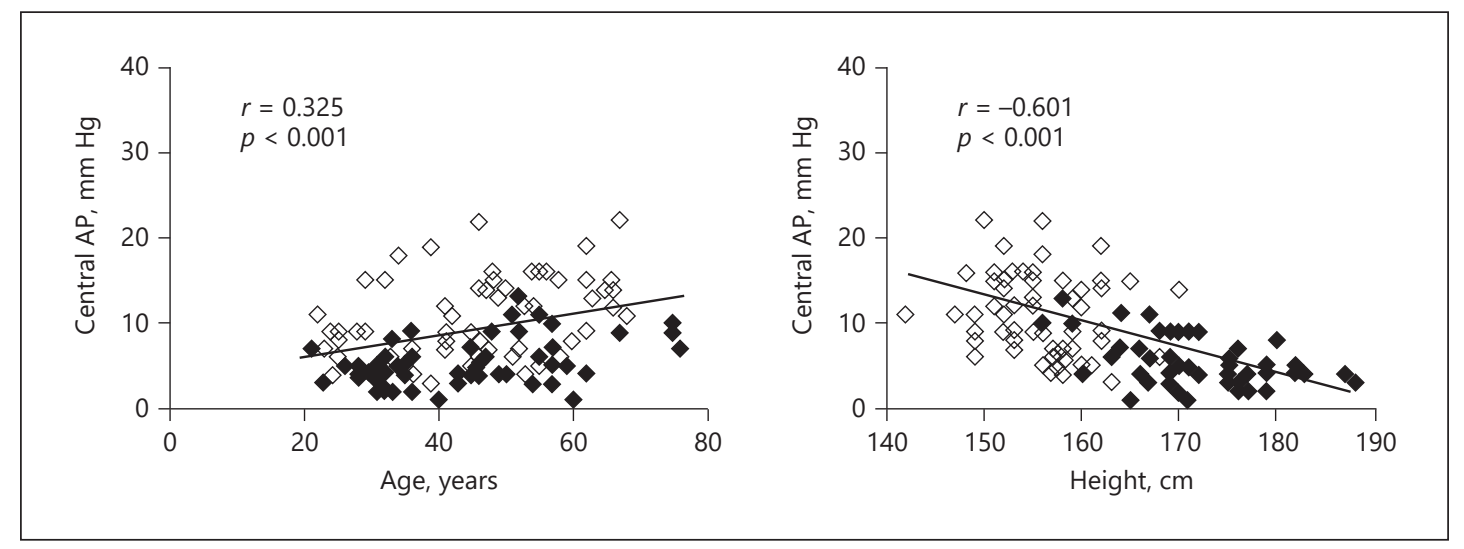

Fig. 1. Pearson correlations between central augmentation pressure (AP) and other variables in the total cohort. The regression line is shown as a solid line. The central AP positively correlated with age in men (black diamond shape; $r=0.408, p=0.003$ ) and women (open diamond shape; $r=0.360, p=0.005$ ).

\section{Results}

Table 1 shows the clinical characteristics and hemodynamic parameters of the participants. We enrolled 110 normotensive volunteers (50 men; age range, 21-76 years). The overall median age of the patients was $44.9 \pm 13.7$ years. No significant difference in age was found between the sexes. Height and weight were greater in men than in women. Brachial mean BP and central systolic BP were higher in men than in women. However, central AIx, central AP, radial AIx, and heart rate were lower in men than in women.

Table 2 shows the Pearson correlations of central AP with other variables using the Mobil-O-Graph. Age, height, and weight were significantly correlated to central AP in the total cohort. Central AP in the total cohort was positively correlated with age $(r=0.325, p<0.001)$ and inversely correlated with height $(r=-0.601, p<0.001)$ (Fig. 1). In multivariate regression analysis, the central AP in the total cohort $\left(R^{2}=0.467\right)$ was significantly associated with age $(\beta=0.097, p<0.001)$, sex $(\beta=-2.890, p=0.010)$, and height $(\beta=-0.153, p=0.031)$.

In the total cohort, the radial AIx in the $\geq 50$-year-old group $(86.6 \pm 10.7 \%)$ significantly increased compared with that in both the 20 - to 39 -year-old group $(67.4 \pm 16.0 \% ; p<0.05)$ 
and the 40- to 49-year-old group $(77.4 \pm 9.4 \% ; p<0.05)$. The central AIx $(27.3 \pm 10.6 \%)$ and central AP (10.0 $\pm 4.8 \mathrm{~mm} \mathrm{Hg})$ in the $\geq 50$-year-old group significantly increased compared with those in the 20- to 39-year-old group (central AIx: $20.0 \pm 10.2 \%, p<0.05$; central AP: 6.7 $\pm 4.2 \mathrm{~mm} \mathrm{Hg}, p<0.05$ ).

\section{Discussion}

This study showed the age-related increase in central AP assessed using the Mobil-OGraph in normotensive Japanese individuals. SphygmoCor is an established device widely used for the estimation of central hemodynamics by radial tonometry [10]. Several factors, including age, sex, height, mean BP, and heart rate, were shown to influence central AP measured by the SphygmoCor in a large number of normotensive European individuals [7]. In this study, age, sex, and height influenced central AP in multivariate regression analysis; however, mean BP and heart rate showed no correlation with central AP.

In the total cohort of this study, no correlation was observed between mean BP and central AP, which may be partially explained by the higher mean BP in men than in women. In contrast, central AP was lower in men than in women. Central AP has been reported to be inversely related to heart rate [11]. In this study, both central AP and heart rate were lower in men than in women, and the age-related decrease in heart rate reported in a large number of normotensive European individuals [7] was not observed (data not shown). These might indicate that central AP was independent of heart rate in the total cohort of this study.

Impedance analysis, based on Fourier-transformed pressure and flow waves, is a valid characterization of the arterial system [12]. Murgo et al. [13] simultaneously measured ascending aortic pressure and flow velocity to analyze ascending aortic impedance and classified the aortic pressure contour to relate the pattern of the impedance spectral plots. The first harmonic of the impedance modulus (Z1) has been recognized as an index of arterial wave reflection [14]. The amplitudes of Z1 were the highest, intermediate, and lowest in type A, B, and C patients, respectively [13]. Central AP is calculated as the increase from the shoulder of the aortic pressure to the late systolic peak in type A and B waveforms or the fall from the initial peak of the aortic pressure to the shoulder in type $C$ waveforms [13]. The inflection point was defined as the zero crossing of the fourth derivative of central aortic pressure [15, 16], and the Mobil-0-Graph uses the fourth derivative rule [6]. Type $C$ waveforms (negative augmentation) are relatively common in younger adults and men. In this study, central AIx and AP were lower in men than in women; however, negative values were not seen. Identification of the inflection point is difficult in type $C$ waveforms [17].

Although the Mobil-O-Graph might overestimate central AP in type C waveforms [5], an age-related increase in central AP was shown in a large European population. Central AP was positively correlated in men $(r=0.37, p<0.001)$ and women $(r=0.35, p<0.001)$ in individuals aged $<30$ to $>80$ years in an Austrian community setting [6]. Similarly, in this study, central AP significantly correlated with age in men $(r=0.408, p=0.003)$ and women $(r=$ $0.360, p=0.005$ ) (Fig. 1). Thus, central AP estimation using the Mobil-0-Graph would be feasible in the Asian population.

The relationship between arterial stiffness and age in a healthy population was assessed in the Framingham Heart Study [18]. It was noted that women had larger reflected waves than men partly due to their shorter height, and height was not sufficient to fully explain the higher reflected wave pressure in women. The main determinant of elevated pulse pressure in women aged $<60$ years in the UK Caucasian population is increased AP, which is related to the mismatch in distal-to-proximal arterial dimensions [19]. 
Recently, the Multi-Ethnic Study of Atherosclerosis showed that short stature was associated with increased risk of incident atherosclerotic cardiovascular disease events [20]. Shorter individuals have been reported to exhibit higher central AIx and AP, and to also have increased risk of coronary artery disease compared with taller individuals in both sexes [21]. In a study in Korean patients with hypertension, female sex and shorter height were shown to be important risk factors of elevated AIx using radial tonometry [4]. The mean height of all Korean patients was $163.4 \pm 8.8 \mathrm{~cm}$, whereas the mean height of the men and women in the Framingham Heart Study was $70 \pm 3$ in. $(177.8 \pm 7.6 \mathrm{~cm})$ and $64 \pm 2$ in. $(162.6 \pm 5.1 \mathrm{~cm})$, respectively. In the present study, the mean height of all the participants was $163.1 \pm 10.1 \mathrm{~cm}$, which is almost similar with the Korean patients. The central AP in all women with a height $\leq 155 \mathrm{~cm}$ was higher than the mean value in men (5.5 mm Hg) (Fig. 1).

There is no single or limited number of discrete reflection sites in the arterial tree, and summation of wave reflection arising from multiple sites reflects central pressure contour [12]. The arrival time of each reflected wave is dependent on aPWV and reflection site distance from the aortic root. As no difference in aPWV was observed between men and women in the present study, a shorter height $\leq 155 \mathrm{~cm}$, as seen in Asian women, would strongly influence central AP. Central AP measurement even for normotensive Asian women might be important to estimate the risk factor of coronary artery disease.

\section{Study Limitations}

The present study has some limitations. First is the lack of invasive measurement of central aortic pressure waveform. Invasive measurement of central aortic pressure waveform is required for high fidelity Millar micromanipulator [22]. However, obtaining ethical approval may be difficult, and it is impossible to perform in many subjects. Second, the population size was relatively small. This study had limited power to detect the curvilinear relationship between central AIx and age. Therefore, we investigated the factors influencing central AP using uni- and multivariate linear regression analyses. Subsequently, rates of carotid-femoral pulse wave velocity (cfPWV) increase have been reported to be accelerated with advancing age in men more than women, leading to a sex-based difference in cfPWV after the age of 50 years [23]. The lack of difference in aPWV between sexes in this study might be due to the algorithm to estimate aPWV by using the Mobil-O-Graph, instead of direct measurement [24]. Finally, these volunteers were almost healthy medical staffs and were not randomly selected from the general population. The results may not reflect the general population.

\section{Conclusions}

In the present study, age-related change in central AP was observed using cuff-based oscillometric measurement. Central AP measurement using the Mobil-0-Graph would be feasible in the Asian population. Normotensive Asian women might have higher reflected wave pressure; therefore, central AP measurement would be important for the estimation of risk factors of coronary artery disease. Further examinations of central hemodynamics in the Asian population are expected.

\section{Acknowledgements}

We thank Dr. Michihiro Kohno for the meaningful discussion as well as Ms. Satomi Kiyota and Ms. Miho Ikeda for their technical assistance. 
Obayashi et al.: Augmentation Pressure by Brachial Oscillometry

\section{Statement of Ethics}

Written informed consent for the procedure was obtained from each volunteer, and the study was approved by the ethics committee of Sanyo-Onoda City Hospital.

\section{Disclosure Statement}

The authors report no specific funding in relation to this research and declare no conflicts of interest.

\section{Author Contributions}

Masakazu Obayashi designed the study, analyzed the data, and wrote the initial draft of the manuscript. Shigeki Kobayashi and Masafumi Yano contributed to data interpretation and assisted in the preparation of the manuscript. All other authors have contributed to data collection and interpretation.

\section{References}

1 Vlachopoulos C, Aznaouridis K, O’Rourke MF, Safar ME, Baou K, Stefanadis C. Prediction of cardiovascular events and all-cause mortality with central haemodynamics: a systematic review and meta-analysis. Eur Heart J. 2010 Aug;31(15):1865-71.

2 Takazawa K, Kobayashi H, Shindo N, Tanaka N, Yamashina A. Relationship between radial and central arterial pulse wave and evaluation of central aortic pressure using the radial arterial pulse wave. Hypertens Res. 2007 Mar;30(3):219-28.

3 Kohara K, Tabara Y, Oshiumi A, Miyawaki Y, Kobayashi T, Miki T. Radial augmentation index: a useful and easily obtainable parameter for vascular aging. Am J Hypertens. 2005 Jan;18(1 Pt 2):11S-4S.

4 Ahn KT, Park KI, Kim MJ, Oh JK, Han JH, Kwon HJ, et al. Height and sex is strongly associated with radial augmentation index in Korean patients with never-treated hypertension. Clin Interv Aging. 2016 Apr;11: 415-22.

5 Wassertheurer S, Kropf J, Weber T, van der Giet M, Baulmann J, Ammer M, et al. A new oscillometric method for pulse wave analysis: comparison with a common tonometric method. J Hum Hypertens. 2010 Aug;24(8): 498-504.

6 Nunan D, Wassertheurer S, Lasserson D, Hametner B, Fleming S, Ward A, et al. Assessment of central haemomodynamics from a brachial cuff in a community setting. BMC Cardiovasc Disord. 2012 Jun;12(1): 48.

7 McEniery CM, Yasmin, Hall IR, Qasem A, Wilkinson IB, Cockcroft JR; ACCT Investigators. Normal vascular aging: differential effects on wave reflection and aortic pulse wave velocity: the Anglo-Cardiff Collaborative Trial (ACCT). J Am Coll Cardiol. 2005 Nov;46(9):1753-60.

8 Weber T, Auer J, O’Rourke MF, Kvas E, Lassnig E, Berent R, et al. Arterial stiffness, wave reflections, and the risk of coronary artery disease. Circulation. 2004 Jan;109(2):184-9.

9 Hametner B, Wassertheurer S, Kropf J, Mayer C, Eber B, Weber T. Oscillometric estimation of aortic pulse wave velocity: comparison with intra-aortic catheter measurements. Blood Press Monit. 2013 Jun;18(3):173-6.

10 Butlin M, Qasem A. Large artery stiffness assessment using SphygmoCor technology. Pulse (Basel). 2017 Jan; 4(4):180-92.

11 Williams B, Lacy PS; CAFE and the ASCOT (Anglo-Scandinavian Cardiac Outcomes Trial) Investigators. Impact of heart rate on central aortic pressures and hemodynamics: analysis from the CAFE (Conduit Artery Function Evaluation) study: CAFE-Heart Rate. J Am Coll Cardiol. 2009 Aug;54(8):705-13.

12 Segers P, O’Rourke MF, Parker K, Westerhof N, Hughes A, Aguado-Sierra J, et al. Towards a consensus on the understanding and analysis of the pulse waveform: Results from the 2016 Workshop on Arterial Hemodynamics: Past, present and future. Artery Res. 2017 Jun;18:75-80.

13 Murgo JP, Westerhof N, Giolma JP, Altobelli SA. Aortic input impedance in normal man: relationship to pressure wave forms. Circulation. 1980 Jul;62(1):105-16.

14 Obayashi M, Yano M, Kohno M, Kobayashi S, Yamamoto T, Ohkusa T, et al. Effect of angiotensin II receptor antagonism on vascular hypertrophy and aortic impedance in abdominal aortic-banded rat. Am J Hypertens. 1999 Apr;12(4 Pt 1):381-7.

15 Kelly R, Hayward C, Avolio A, O’Rourke M. Noninvasive determination of age-related changes in the human arterial pulse. Circulation. 1989 Dec;80(6):1652-9.

16 Gatzka CD, Kingwell BA, Cameron JD, Berry KL, Liang YL, Dewar EM, et al.; ANBO2 investigators. Australian Comparative Outcome Trial of Angiotensin-Converting Enzyme Inhibitor- and Diuretic-Based Treatment of Hypertension in the Elderly. Gender differences in the timing of arterial wave reflection beyond differences in body height. J Hypertens. 2001 Dec;19(12):2197-203. 
17 Hughes AD, Park C, Davies J, Francis D, McG Thom SA, Mayet J, et al. Limitations of augmentation index in the assessment of wave reflection in normotensive healthy individuals. PLoS One. 2013;8(3):e59371.

18 Mitchell GF, Parise H, Benjamin EJ, Larson MG, Keyes MJ, Vita JA, et al. Changes in arterial stiffness and wave reflection with advancing age in healthy men and women: the Framingham Heart Study. Hypertension. 2004 Jun;43(6):1239-45.

19 Cecelja M, Jiang B, McNeill K, Kato B, Ritter J, Spector T, et al. Increased wave reflection rather than central arterial stiffness is the main determinant of raised pulse pressure in women and relates to mismatch in arterial dimensions: a twin study. J Am Coll Cardiol. 2009 Aug;54(8):695-703.

20 Yeboah J, Blaha MJ, Michos ED, Qureshi W, Miedema M, Flueckiger P, et al. Adult height, prevalent coronary artery calcium score, and incident cardiovascular disease outcomes in a multiethnic cohort. Am J Epidemiol. 2017 Oct; 186(8):935-43.

21 Reeve JC, Abhayaratna WP, Davies JE, Sharman JE. Central hemodynamics could explain the inverse association between height and cardiovascular mortality. Am J Hypertens. 2014 Mar;27(3):392-400.

22 O'Rourke MF, Kim M, Adji A, Nichols WW, Avolio A. Use of arterial transfer function for the derivation of aortic waveform characteristics. J Hypertens. 2004 Feb;22(2):431-2.

23 AlGhatrif M, Strait JB, Morrell CH, Canepa M, Wright J, Elango P, et al.; The Baltimore Longitudinal Study of Aging. Longitudinal trajectories of arterial stiffness and the role of blood pressure: the Baltimore Longitudinal Study of Aging. Hypertension. 2013 Nov;62(5):934-41.

24 Grillo A, Parati G, Rovina M, Moretti F, Salvi L, Gao L, et al. Short-term repeatability of noninvasive aortic pulse wave velocity assessment: comparison between methods and devices. Am J Hypertens. 2017 Dec;31(1):80-8. 